\title{
A IMPORTÂNCIA DA ESPÉCIE Mussismilia braziliensis PARA O PARQUE NACIONAL MARINHO DOS ABROLHOS
}

\author{
Alexsandro Santos da Silva ${ }^{1}$ \\ Juzenilda Gomes Figuerêdo ${ }^{2}$
}

\begin{abstract}
Resumo: A espécie Mussismilia braziliensis (Verrill 1868), endêmica do banco dos Abrolhos, teve grande importância para a criação do Parque Nacional Marinho dos Abrolhos (ParNaM-Abrolhos). Com objetivo de apresentar os impactos que atingem a espécie e, descrever os trabalhos realizados nesta unidade que versam a temática Educação Ambiental (EA), foi realizado revisão de literatura sobre o tema e o estágio voluntário na unidade. O branqueamento, praga branca e banda negra são algumas das problemáticas que af etam a espécie estudada, sendo que a sobrepesca, mu danças climáticas, degradação da zona costeira, turismo, poluição em decorrência da instalação de projetos industriais, a exploração de petróleo, variações do nível do mar, algumas das ações que têm ameaçados os corais. Dentre os trabalhos de EA destacam-se: Programa Voluntariado, Abra os olhos para a Ciências, Comunidade em Abrolhos e a Conduta Consciente, que são alguns dos programas existentes nesta unidade. Por fim, os trabalhos desenvolvidos em EA no ParNaM-Abrolhos são importantes, pois auxiliam na formação de cidadãos conscientes, visando a importância dos corais existentes no Banco dos Abrolhos, inclusive, a espécie foco do estudo para equilíbrio ambiental da Unidade de Conservação.
\end{abstract}

Palavras-chave: Endêmico, Preservação, Complexo Recifal dos Abrolhos.

Abstract: The species Mussismilia braziliensis (Verrill 1868), endemic to the Abrolhos bank, was of great importance for the creation of the Abrolhos National Marine Park (ParNaMAbrolhos). In order to present the impacts that affect the species and to describe the works carried out in this unit that deal with the Environmental Education (EA) theme, a literature review on the theme and the voluntary internship in the unit was carried out. Whitening, white plague and black band are some of the problems that affect the species studied, being that overfishing, climate change, degradation of the coastal zone, tourism, pollution due to the installation of industrial projects, oil exploration, variations in the level of the sea, some of the actions that have threatened the corals. Among the EE work, the following stand out: Volunteer Program, Open your eyes to Science, Community in Abrolhos and Conscious Conduct, which are some of the existing programs in this unit. Finally, the works developed in EE at ParNaM-Abrolhos are important, as they assist in the formation of conscious citizens, aiming at the importance of the existing corals at Banco dos Abrolhos, including the species that is the focus of the study for environmental balance of the Protect Area.

Keywords: Endemic, Preservation, Abrolhos Reef Complex.

${ }^{1}$ Universidade do Estado da Bahia.E-mail:lex-1alex@hotmail.com

2 Universidade do Estado da Bahia.E-mail:jfigueredo@uneb.br 


\section{Introdução}

O complexo recifal de Abrolhos engloba a mais extensa área de recifes coralíneos de todo o oceano Atlântico Sul, possuindo menos de $1 \%$ dos ambientes recifais do planeta Terra. Estes recifes são raros no mundo, além de apresentarem uma incontestável importância científica (LEÃO, 2002, p.346).

Devido a sua beleza cênica, variedades, formas e cores, os recifes de corais em todo o mundo tornam-se conhecidos, além de chamar atenção para seu valor econômico e até mesmo forte atração para o turismo. Nos recifes brasileiros, os do Banco dos Abrolhos são os mais exuberantes, nesta região são encontradas espécies de corais endêmicas do Brasil (SILVA, 2005, p.223).

A espécie Mussismilia braziliensis (VERRILL 1868) conhecida como coral cérebro, é uma espécie de coral pétreo, endêmica da região de Abrolhos (PÁDUA; CAVALCANTI, 2012, p.1), é hermafrodita e desovadora de gametas (CAPARELLI, 2004, p.44). Os seus pólipos são cilíndricos, possuindo uma extremidade oral (boca e tentáculos) (RUPPERT; BARNES, 1996 Apud SABADINI, 2017, pg.19), encontrada preferencialmente em águas limpas e rasas, locais que possuem maior penetração de luz (DUTRA, 2003, p.16).

Este trabalho tem como base apresentar os impactos que atingem a espécie de coral, M. braziliensis, residente no Parque Nacional Marinho dos Abrolhos (ParNaM-Abrolhos), além de descrever os trabalhos realizados nesta unidade que versam a temática Educação Ambiental (EA).

\section{Metodologia}

Foi feito um levantamento bibliográfico dando ênfase aos trabalhos com a espécie $M$. braziliensis e que retratam as atividades de EA desenvolvidas no ParNaM-Abrolhos.

Buscando conhecer e ter acessos aos trabalhos de EA desenvolvidos no ParNaM-Abrolhos, além do levantamento bibliográfico, foi necessário a realização do estágio na unidade, através do Programa Voluntariado. Este, é um programa do Instituto Chico Mendes de Conservação da Biodiversidade ICMBIO, que tem como objetivo incentivar o ser humano na preservação e conservação da biodiversidade por meio do trabalho sem fins lucrativos.

\section{Resultados e discussão}

\section{Histórico do Parque Nacional Marinho dos Abrolhos}

Devido às degradações que o mar vem sofrendo, por depredações e por contaminações resultantes da ação humana, pesca indiscriminada, coleta de espécimes, como as estrelas do mar e os corais para uso artesanais, além de vários outros fatores abusivos contra áreas marinhas, foi pensado na criação de Unidade de Conservação marinha, não somente no Brasil, mas em todo o mundo (IBAMA, 1991, p.10). 
Os primeiros movimentos favoráveis à criação do primeiro Parque Nacional Marinho do Brasil vieram dos pesquisadores da Universidade Federal da Bahia (UFBA), do Museu Nacional do Rio de Janeiro e da Universidade de São Paulo (USP) (CAPELAS JUNIOR, 2013, p.42). Após estes movimentos para a criação da Unidade de Conservação, os pesquisadores da UFBA apresentaram ao público informações sobre o arquipélago dos Abrolhos, contribuindo para o conhecimento da área (IBAMA, 1991, p.11).

Em 1983, atendendo aos apelos dos pesquisadores, além de outros conservacionistas, o Ministério da Marinha e da Agricultura enviaram ao presidente da República o primeiro esboço do decreto de criação juntamente com as justificativas e os objetivos. Assim, em 6 de Abril de 1983 foi criado em Abrolhos o primeiro parque nacional marinho brasileiro, tendo à assinatura do decreto de número 88.218 (CAPELAS JUNIOR, 2013, p.42).

A área do parque constitui 4 ilhas (Redonda, Siriba, Sueste e Guarita), além do parcel dos Abrolhos e do recife das Timbebas, totalizando cerca de 98 mil hectares descontínuos (ICMBIO, 2019³).

\section{Trabalhos realizados no Parque Nacional Marinho Dos Abrolhos}

Ao longo das décadas foram realizados diversos trabalhos abordando vários temas no ParNaM- Abrolhos, segundo RANIERIE; ROSAMIGLIA (2007, p.72) em 1988 esta unidade inicia suas atividades com as Instituições parceiras tendo como objetivo a conscientização dos visitantes.

Entre 1988 a 2000 os trabalhos de conscientização em relação às questões ambientais continuaram. No ano de 2000 , o parque recebeu a visita de 73 alunos do Centro Educacional Da Vinci de Vitória do Espírito Santo, no qual foi possível ser transmitidos orientações socioambientais sobre os ambientes recifais. Com eles vieram também um médico e professores de Natação, Educação Física e Biologia (CENTRO EDUCACIONAL LEONARDO DA VINCI, $2000)^{4}$.

Em 2001 foi elaborada a Campanha da conduta consciente em ambientes recifais com o objetivo de sensibilizar os moradores das comunidades do entorno e demais, dando ênfase a importância de conservar e preservar estes ambientes (IBAMA, 2009).

\footnotetext{
3 Instituto Chico Mendes de Conservação da Biodiversidade. Quem somos. Disponível em: http://www.icmbio.gov.br/parnaabrolhos/quem-somos.html. Acesso em: 29 de Set. de 2019 ${ }^{4}$ Centro Educacional Leonardo Da Vinci. Viagem Acadêmica. Disponível em: http://www2.davincivix.com .br/davinci.php?area=noticias\&e=61\&c=man4aea39ff994132227. Acesso em: 15 de Ago. de 2015.
} 
Desde 2002 acontece o Projeto de Monitoramento de Recifes de Coral no Parque Nacional Marinho de Abrolhos, verificando o estado de conservação deste ambiente rico em endemismo (ICMBIO, 2015) .

Em 2003 foi implantado o Programa de Educação Ambiental (EA). A partir daí surgiram seguimentos como, a implantação e estruturação do Núcleo de Educação Ambiental (NEA), além do projeto "Olhos Abertos" (RANIERIE; ROSAMIGLIA, 2007, p.73). No ano seguinte, em 2004, o NEA implanta o seu programa de Estágio Voluntário, tendo nesse mesmo ano, a capacitação e treinamento de dois estagiários (IBAMA, 2006, p.23).

No ano de 2005, através do Programa Professores no Parque, realizouse oficinas de Capacitação para os professores de Caravelas. No mesmo ano, foram ministradas palestras nas escolas e nas colônias de pescadores do entorno do parque (RANIERIE; ROSAMIGLIA, 2007, p.75).

Em 2006, através do programa "Professores no Parque", foi proporcionado a dez professores da rede Municipal de Ensino da cidade Nova viçosa, um curso de capacitação com foco de aperfeiçoar estes professores para a área de EA (PREFEITURADE NOVA VIÇOSA, 2009) ${ }^{6}$.

No ano de 2007 foi desenvolvido no ParNaM-Abrolhos, pela Universidade Estadual de Maringá (UEM) e a Cl-Brasil, o projeto "Abra os Olhos para a Ciência" com o objetivo de incentivar os alunos do Colégio Polivalente de Caravelas a desenvolver habilidade científica para a resolução dos problemas socioambientais (CONSERVAÇÃO INTERNACIONAL, 2015) ${ }^{7}$.

Em 2008 o ParNaM- Abrolhos apoiou um evento com o intuito de conscientizar a comunidade vizinha sobre as questões ambientais e culturais da região, desenvolvido com parceria do Projeto Meros do Brasil e o Movimento Cultural ArteManha. Este evento ocorreu através das execuções de atividades de educação Ambiental, além de constar com atrativos culturais, oficinas de dança, coordenadas pelo Movimento Cultural ArteManha (TV ECOLÓGICA, 2008) $)^{8}$

Com o intuito de envolver e sensibilizar o público que vive na área do entorno do parque, foi criado em 2009 o programa Comunidade em Abrolhos

\footnotetext{
${ }^{5}$ Instituto Chico Mendes de Conservação da Biodiversidade. Projeto de Monitoramento de Recifes de Coral. Disponível em: http://www.icmbio.gov.br/portal/o-que-fazemos/pesquisa-emonitoramento/monitoramento/programas-de-monitoramento.html. Acesso em: 20 de Set. de 2015 ${ }^{6}$ Prefeitura de Nova Viçosa. Programa Professores no Parque. Disponível em: http://www.novavicosa.ba.gov.br/modules/news/index.php?start=1170\&storytopic=0 . Acesso em: $22 \mathrm{de}$ Set. de 2015

${ }^{7}$ Conservação Internacional. Iniciativas em Abrolhos Terra e Mar. Disponível em: http://www.conservation.org/global/brasil/iniciativas-atuais/Pages/iniciativas-em-abrolhos-terra-e-mar.aspx. Acesso em: 20 de Jul. de 2015

8 TV Ecológica. Festa da Baleia. Disponível em: https://tvecologica.wordpress.com/2008/07/23/. Acesso em: 03 de Out. de 2015
}

Revbea, São Paulo, V. 15, № 1: 351-359, 2020. 
(ICMBIO, 2015) ${ }^{9}$. No ano seguinte, em 2010, foi criado o programa Criança no Parque, tendo como objetivo sensibilizar e formar cidadãos conscientes dando relevância a preservação do Meio Ambiente e, com esse objetivo, em 2011 foi realizado pela segunda vez o evento "Crianças no Parque", tendo cerca de 100 crianças da Escola Municipal Afrízio Vieira Lima, da comunidade da Barra de Caravelas (UNIDADE DE CONSERVAÇÃO, 2011) ${ }^{10 .}$

Além do Criança no Parque, foi iniciado em 2011 o Programa de Voluntariado, regulamentado a partir da Instrução Normativa $n^{\circ}$ 03/2009 com o objetivo de incentivar a participação da sociedade e aproximá-la da gestão das áreas protegidas e da conservação da biodiversidade (ICMBIO, 2015) ${ }^{11}$.

Em 2012 com parceria a Associação de Estudos Costeiros e Marinhos dos Abrolhos - ECOMAR e as Secretarias de Educação e Meio Ambiente dos municípios de Caravelas, Alcobaça, Prado, Mucuri e Nova Viçosa, foi realizado para 40 professores e coordenadores pedagógicos da rede pública de ensino, alunos de graduação da Universidade do Estado da Bahia - UNEB, além de técnicos das Secretarias Municipais de Meio Ambiente e Educação, o Programa Professores no Parque (ICMBIO, 2015) ${ }^{12}$.

Em 2013 foi realizado o Programa de Monitoramento Recifal do ICMBio no ParNaM- Abrolhos. Este monitoramento acontece com base na metodologia aplicada pelo programa Reef Check, este é um programa internacional de monitoramento dos recifes de coral (ICMBIO, 2013) ${ }^{13 .}$

Em 2014 o Movimento Cultural Artemanhajuntamente coma Organização não governamental (ONG) Patrulha Ecológica desenvolveu no Centro de Visitantes do ParNaM-Abrolhos, atividades de EA e Cultural, buscando estimular o interesse dos alunos da Escola Municipal Ede Santos e demais visitantes para as questões históricas, socioambientais e cultu rais da região (COELHO, 2014) ${ }^{14}$.

Em 2019 foram oportunizadas 110 vagas, para que grupos tenham acesso ao ParNaM-Abrolhos, além de desfrutar momentos de lazer, valorizando a importância da conservação da biodiversidade e o desenvolvimento

\footnotetext{
${ }^{9}$ Instituto Chico Mendes de Conservação da Biodiversidade. Comunidade em Abrolhos. Disponível em: http://www.icmbio.gov.br/parnaabrolhos/destaques/20-programa-comunidade-em-abrolhos.html. Acesso em: 29 de Jul. de 2015

10 Unidade de Conservação. Programa Criança no Parque. Disponível em:

http://uc.socioambiental.org/noticia/parque-nacional-marinho-dos-abrolhos-comemora-o-dia-das-criancas. Acesso em: 15 de Out. de 2015

${ }^{11}$ Instituto Chico Mendes de Conservação da Biodiversidade. Guia do Voluntário. Disponível em: http://www.icmbio.gov.br/parnaabrolhos/seja-um-voluntario. Acesso em: 20 de Out. de 2015

${ }_{12}$ Instituto Chico Mendes de Conservação da Biodiversidade. Programa Professores no Parque.

Disponível em: http://www.icmbio.gov.br/parnaabrolhos/destaques/26-programa-professores-no-parque2012.html. Acesso em: 12 de Jul. de 2015

${ }^{13}$ Instituto Chico Mendes de Conservação da Biodiversidade. Campanha de Monitoramento. Disponível em: http://www.icmbio.gov.br/portal/comunicacao/noticias/20-geral/4566-abrolhos-realiza-segundacampanha-de-monitoramento-de-corais-de-2013.html. Acesso em: 23 de Set. de 2015

${ }^{14}$ Coelho, Adriene. Farol para Abrolhos. Disponível em:

http://farolparaabrolhos.blogspot.com.br/2014 0401 archive.html. Acesso em: 20 de Set. de 2015
}

Revbea, São Paulo, V. 15, № 1: 351-359, 2020 
sustentável na região, a grupos do Território da Costa das Baleias, através do edital Comunidade em Abrolhos. Neste mesmo ano, foi aberto o edital Curso de Formação de Condutores de Visitantes da Unidade, profissionais estes, que desenvolvem atividades informativas e interpretativas sobre 0 ambiente, garantindo a segurança dos mesmos e a proteção dos ambientes naturais utilizados (ICMBIO, 2019).

Nos últimos anos, vários projetos foram desenvolvidos na tentativa de aproximar o Parque, que é geograficamente distante da população local (BARROS, 2016, pg.114), além de conservar e preservar os ecossistemas recifais. Dentre os projetos destacam-se: Programa Voluntariado, Abra os olhos para a Ciências, Comunidade em Abrolhos e a Conduta Consciente, que são alguns dos programas existentes.

Desde 1987 até 2019 as atividades de EA, através de palestras com a comunidade escolar e demais comunidades circunvizinhas, além de acesso à Unidade, tendo como objetivo primordial preservação e conservação da região, dentre tantas outras atividades, continuaram. Neste trabalho foi apresentada uma coletânea com as principais atividades que o Parque tem e vem realizando ao longo de sua história de criação.

\section{Impactos que ameaçam a espécie M. braziliensis}

Através das observações realizadas com o programa voluntariado foi possível perceber a grande importância que a espécie $M$. braziliensis tem para o Parque Nacional Marinho dos Abrolhos. Portanto, para avaliar a relação de residência da espécie $M$. braziliensisnesta unidade de conservação, onde temos recifes de corais no entorno das ilhas, foi observado que esta espécie é a principal na construção dos recifes neste local. De acordo com Moura (2014), os principais construtores recifais são considerados espécies-relíquia, espécies de corais mais antigas que sobrevivem apenas em alguns locais da costa brasileira.

A espécie $M$. braziliensis está correndo sério risco de entrar em extinção, devido a várias ameaças que este coral, assim como outros, estar suscetível (PÁDUA; CAVALCANTI, 2012, p.1). Um dos eventos que mais está afetando os corais é o branqueamento que pode ser definido como a perda da pigmentação, ou seja, perda de algas simbiontes. Esta simbiose é responsável pela coloração dos corais, e a quebra desta relação provoca a despigmentação da colônia, levando ao branqueamento (LEÃO, 1996; MIGOTTO et al. 1997; CORNELL; KARLSON, 2000; DULLO, 2005 apud SOARES; RABELO, 2014 p.2), podendo significar sérios danos aos corais, inclusive a espécie estudada.

Esta espécie está diante de duas grandes ameaças que possibilita a extinção deste, que são os agentes antropogênicos e os agentes naturais, sendo que o ParNaM-Abrolhos, por mais que seja uma unidade protegida da ação humana, ainda assim a $M$. braziliensis, as demais espécies de corais e outros organismos, estão sujeitas a diversas ameaças antrópicas e naturais. 
problemáticas que podem ocasionar a morte desta espécie, sendo o aquecimento global e à degradação da zona costeira, fatores que podem estar provocando estas doenças (PÁDUA; CAVALCANTI, 2012, p.3)

O turismo, a exploração de recursos naturais, a poluição em decorrência da instalação de projetos industriais e a exploração de petróleo são algumas das ações que têm ameaçado os recifes de Abrolhos (AMADO FILHO et al. 1997; COUTINHO et al. 1993, LEÃO, 1994 1996; LEÃO, et al. 1994 apud LEÃO, 2002, p.357). Mesmo que os três últimos impactos citados, decorrentes à ação humana, não ocorrem dentro da unidade de conservação, porém podem prejudicar os recifes de corais do ParNaM-Abrolhos, já que esta unidade fica cerca de $70 \mathrm{~km}$ da zona costeira.

A exploração de petróleo no litoral da Bahia, negociada pelo governo, põe em risco a região do Banco dos Abrolhos, área reconhecida pela riqueza da biodiversidade ímpar no Atlântico Sul (WWF-BRASIL, 2019).

De acordo com Freitas et al $(2012$, p.143) os recifes de corais também estão ameaçados por causa das mudanças climáticas, variações do nível do mar, explosão populacional de predadores, doenças e branqueamento dos corais.

A inexistência de peixes herbívoros, outra problemática, ocasionaria o elevado crescimento das algas, consequentemente, o sufocamento dos corais. Portanto, a sobrepesca no banco dos Abrolhos, além de pressionar as espéciesalvo, pode acarretar um desequilíbrio ambiental, já que sem os peixes, principalmente os herbívoros, a vida dos corais estaria comprometida (MOURA, 2014). A partir disso, é possível entender a importância que esta espécie tem para o Parque Nacional Marinho dos Abrolhos.

A atividade turística na região de Abrolhos tem aumentado na última década, exercendo uma grande pressão no ecossistema recifal (SPANO et al. 2008, p.1). Mesmo sendo essa atividade uma das ameaças, no turismo pode ocorrer a prática de Educação Ambiental, pois contribui para a transformação do comportamento de todos os envolvidos, sendo forte agente sensibilizador das questões socioambientais (GERLING; SILVA JUNIOR, 2016, p.48).

\section{Considerações finais}

O coral $M$. braziliensis é uma espécie de coral cérebro de grande relevância para o ParNaM-Abrolhos, apresentando uma incontestável importância para a manutenção do equilíbrio ecológico, sendo a principal espécie na construção dos recifes de corais da região. Este coral destacou-se como umas das espécies decisivas para a criação desta unidade de conservação, pois além de estar sendo ameaçada de extinção, é somente encontrada nesta imensa área denominada Banco dos Abrolhos. 
É necessário conservar o ecossistema ao qual se encontra a $M$. braziliensis e demais organismos, porém é preocupante a falta de conscientização da humanidade que vem degradando os recifes, prejudicando não somente esta espécie, mas também tantas outras.

Por fim, os trabalhos desenvolvidos em EA no ParNaM-Abrolhos são importantes, pois auxiliam na formação de cidadãos conscientes, visando a importância dos corais existentes no Banco dos Abrolhos, inclusive, a espécie foco do estudo para equilíbrio ambiental da unidade.

\section{Agradecimentos}

Ao Parque Nacional Marinho dos Abrolhos e a equipe de monitores da Unidade, pelo apoio à pesquisa.

\section{Referências}

BARROS, J. G. R. Abr'olhos! Uma análise histórica do Parque Nacional Marinho dos Abrolhos com as comunidades pesqueiras de Caravelas, BA / Janaína Gonçalves Rios Barros. - Viçosa, MG. 2016. Disponível em: $<$ https://www.locus.ufv.br/bitstream/handle/123456789/10053/texto\%20complet o.pdf?sequence=1\&isAllowed=y $>$ Acesso em: 20 de Jul. 2017.

CAPARELLI, A. C. PIRES, D. O.; JUNQUEIRA, A. O. R.; PITOMBO, F. B. Esforço reprodutivo de Mussismilia braziliensis (Verrill, 1868) (Cnidaria, Scleractinia, Mussidae) no Complexo Recifal dos Abrolhos, BA, Brasil. 2004. Dissertação (Mestrado em Ciências Biológicas (Zoologia)) - Universidade Federal do Rio de Janeiro, 2004.

DUTRA, L. X. C. Os efeitos do aporte de sedimento na vitalidade dos recifes da região de Abrolhos, Bahia. 2003. $84 \mathrm{f}$. Dissertação (Mestrado). Instituto de Geociências, Universidade Federal da Bahia, Salvador, 2003.

FREITAS, L. M.; OLIVEIRA, M. D. M.; KIKUCHI, R. K. P.; KIKUCHI, R. K. P. Os mecanismos de sobrevivência dos corais diante do impacto das mudanças climáticas sobre o ecossistema de recifes. 2012. Cadernos de Geociencias (UFBA), v. 9, p. 142-156, 2012.

GERLING, C.; SILVA JUNIOR, J. M. Desafios Para Conservação: As Faces Do Turismo. 2016. In: GERLING, C.; RANIERE, C.; FERNANDES, L.; GOUVEIA M.T.J.; ROCHA V. (Org.). Manual De Ecossistemas Marinhos E Costeiros Para Educadores. 1ed.Santos: Editora Comunicar, v.1, P. 48-48. 2016.

IBAMA (Instituto Brasileiro do Meio Ambiente e dos Recursos Naturais Renováveis). Conduta consciente em ambientes recifais. 2009. Gerência de Biodiversidade Aquática e Recursos Pesqueiros. - Brasília: MMA/SBF. 2009.

IBAMA (Instituto Brasileiro do Meio Ambiente e dos Recursos Naturais Renováveis), Curso de Capacitação Continuada de Formação de Condutores para o Centro de Visitantes. 2006. IBAMA, Brasília, 2006. 
IBAMA (Instituto Brasileiro do Meio Ambiente e dos Recursos Naturais Renováveis), Plano de manejo do Parque Nacional Marinho de Abrolhos. 1991. IBAMA, Brasília. 1991.

ICMBIO (Institu to Chico Mendes de Conservação da Biodiversidade). Download de Arquivos. 2019.2 Disponível em: $<$ http://www.icmbio.gov.br/parnaabrolhos/downloads.html> Acesso em: Acesso em: 20 de Dez. de 2019.

CAPELASJÚNIOR, A. Abrolhos. Editora Cultura Sub, São Paulo, 2013.

LEÃO, Z.M.A.N. Abrolhos, BA - O complexo recifal mais extenso do Atlântico Sul. 2002. In: SCHOBBENHAUS, C.; CAMPOS, D.A.; QUEIROZ, E.T.; WINGE, M.; BERBERT-BORN, M.L.C. (Edits.) Sítios Geológicos e Paleontológicos do Brasil. 1. ed. Brasilia: DNPM/CPRM - Comissão Brasileira de Sítios Geológicos e Paleobiológicos (SIGEP), v.01: 345-359. 2002.

MOURA, R.L. D. Unidades de Conservação no Brasil/Institu to Socioambiental. 2014. Disponível em:<https://uc.socioambiental.org/en/noticia/135158 >Acesso em: Acesso em: 20 de Jul. de 2015.

PADUA, A.P.S.L.D.E; CAVALCANTI, J.S.S. Análise sobre as causas das doenças em Mussismilia braziliensis (Verrill 1868) no Banco de Abrolhos: Uma Revisão Bibliográfica. 2012. Anais do Congresso Brasileiro de Oceanografia. 2012. Disponível em: <http://users5.nlink.com.br/ diariodeummuseu/wordpress/wpcontent/uploads/2011/09/0597.pdf ana.pdf>. Acesso em: 15 de Jan. de 2015.

RANIERI, C. L.; ROSAMIGLIA, P. R. F. Parque Nacional Marinho dos Abrolhos: Núcleo de educação ambiental. 2007. In: NEIMAN, Z.; JUNQUEIRA, V. (Org.). Educação Ambiental e Conservação da Biodiversidade. Barueri, SP: Manole, p.67-79, 2007.

SABADINI, S. D. C. Suscetibilidade dos recifes de Abrolhos aos eventos de branqueamento de corais. 2017. Dissertação (Mestrado), Instituto de Geociências, Universidade Federal de Minas Gerais. Belo Horizonte, p. 89, 2017.

SILVA, M. Apresentação. 2005 In: Megadiversidade, p 223. 2005.

SOARES, M. O.; RABELO, E. F. Primeiro registro de branqueamento no litoral do Ceará (NE-Brasil): Indicador das mudanças climáticas? Geociências (São Paulo. Online), v. 33, p. 1-10, 2014.

SPANÓ, S.; LEÃO, Z. M. A. N.; Kikuchi, R. K. P. Diagnóstico do estado de conservação dos recifes em franja do Parque Nacional Marinho dos Abrolhos. 2008. OLAM: Cienc. Tecnol., v. 8, n. 2, p. 245-277, 2008.

WWW-BRASIL. Ameaçada, biodiversidade sustenta a economia na região dos Abrolhos. 2019. Disponível em: $<$ https://www.wwf.org.br/?73343/Ameacada-biodiversidade-sustenta-aeconomia-na-regiao-dos-Abrolhos >. Acesso em: 15 de NOV. de 2019. 